\title{
Solvability of the Brinkman-Forchheimer-Darcy Equation
}

\author{
Piotr Skrzypacz and Dongming Wei \\ School of Science and Technology, Nazarbayev University, 53 Kabanbay Batyr Ave., Astana 010000, Kazakhstan \\ Correspondence should be addressed to Piotr Skrzypacz; piotr.skrzypacz@nu.edu.kz
}

Received 26 May 2017; Accepted 19 June 2017; Published 20 July 2017

Academic Editor: Malgorzata Peszynska

Copyright (C) 2017 Piotr Skrzypacz and Dongming Wei. This is an open access article distributed under the Creative Commons Attribution License, which permits unrestricted use, distribution, and reproduction in any medium, provided the original work is properly cited.

\begin{abstract}
The nonlinear Brinkman-Forchheimer-Darcy equation is used to model some porous medium flow in chemical reactors of packed bed type. The results concerning the existence and uniqueness of a weak solution are presented for nonlinear convective flows in medium with variable porosity and for small data. Furthermore, the finite element approximations to the flow profiles in the fixed bed reactor are presented for several Reynolds numbers at the non-Darcy's range.
\end{abstract}

\section{Introduction}

In this section we introduce the mathematical model describing incompressible isothermal flow in porous medium without reaction. The considered equations for the velocity and pressure fields are for flows in fluid saturated porous media. These problems are of importance for example in oil reservoir optimization; see [1]. Most research results for flows in porous media are based on the Darcy equation which is considered to be a suitable model at a small range of Reynolds numbers. However, there are restrictions of Darcy equation for modelling some porous medium flows; that is, in closely packed media, saturated fluid flows at slow velocity but with relatively large Reynolds numbers. The flows in such closely packed medium behave nonlinearly and cannot be modelled accurately by the Darcy equation which is linear. The deficiency can be circumvented with the Brinkman-Forchheimer-Darcy law for flows in closely packed media, which leads to the following model: let $\Omega \subset$ $\mathbb{R}^{n}, n=2,3$, represent the reactor channel. We denote its boundary by $\Gamma=\partial \Omega$. The conservation of volume-averaged values of momentum and mass in the packed reactor reads as follows

$$
\begin{aligned}
-\operatorname{div}(\varepsilon \nu \nabla \mathbf{u}-\varepsilon \mathbf{u} \otimes \mathbf{u})+\frac{\varepsilon}{\varrho} \nabla p+\sigma(\mathbf{u}) & =\mathbf{f} \quad \text { in } \Omega, \\
\operatorname{div}(\varepsilon \mathbf{u}) & =0 \quad \text { in } \Omega,
\end{aligned}
$$

where $\mathbf{u}: \Omega \rightarrow \mathbb{R}^{n}$ and $p: \Omega \rightarrow \mathbb{R}$ denote the unknown velocity and pressure, respectively. The positive quantity $\varepsilon=\varepsilon(\mathbf{x})$ stands for porosity which describes the proportion of the nonsolid volume to the total volume of material and varies spatially in general. The expression $\sigma(\mathbf{u})$ represents the friction forces caused by the packing of spherical particles with one constant diameter. The right hand side $\mathbf{f}$ represents an outer force (e.g., gravitation), $\varrho$ the constant fluid density, and $v$ the constant kinematic viscosity of the fluid, respectively. The expression $\mathbf{u} \otimes \mathbf{u}$ symbolizes the dyadic product of $\mathbf{u}$ with itself.

The formula given by Ergun [2] will be used to model the influence of the packing on the flow inertia effects

$$
\sigma(\mathbf{u})=150 \nu \frac{(1-\varepsilon)^{2}}{\varepsilon^{2} d_{p}^{2}} \mathbf{u}+1.75 \frac{1-\varepsilon}{\varepsilon d_{p}} \mathbf{u}|\mathbf{u}| .
$$

Thereby $d_{p}$ stands for the diameter of pellets and $|\cdot|$ denotes the Euclidean vector norm. The linear term in (2) accounts for the head loss according to Darcy and the quadratic term is the Forchheimer law. The model (1)-(2) with the pressure term of the form $\nabla(\varepsilon p)$ has been proposed in [3, Section 2.2]. For the derivation of the equations, their limitations, modelling, and homogenization questions in porous media we refer to [4-9] and [10, Chapter 1].

To close the system (1) we prescribe Dirichlet boundary condition

$$
\left.\mathbf{u}\right|_{\Gamma}=\mathbf{g}
$$


where

$$
\int_{\Gamma_{i}} \varepsilon \mathbf{g} \cdot \mathbf{n} d s=0
$$

has to be fulfilled on each connected component $\Gamma_{i}$ of the boundary $\Gamma$. The distribution of porosity $\varepsilon$ is assumed to satisfy the bounds

$$
0<\varepsilon_{0} \leq \varepsilon(\mathbf{x}) \leq \varepsilon_{1} \leq 1 \quad \forall \mathbf{x} \in \Omega,
$$

with some constants $0<\varepsilon_{0}, \varepsilon_{1} \leq 1$.

A comprehensive account of fluid flows through porous media beyond Darcy law's valid regimes and classified by the Reynolds number can be found in, for example, [11]. Also, see [12] for simulating pumped water levels in abstraction boreholes using such nonlinear Darcy-Forchheimer law and [13-15] for recent references on this model.

In the next section we use the porosity distribution which is estimated for packed beds consisting of spherical particles and takes the near wall channeling effect into account. This kind of porosity distribution obeys assumption (A1).

Let us introduce dimensionless quantities

$$
\begin{aligned}
& \mathbf{u}^{*}=\frac{\mathbf{u}}{U_{0}}, \\
& p^{*}=\frac{p}{\varrho U_{0}^{2}}, \\
& \mathbf{x}^{*}=\frac{\mathbf{x}}{d_{p}}, \\
& \mathbf{g}^{*}=\frac{\mathbf{g}}{U_{0}},
\end{aligned}
$$

where $U_{0}$ denotes the magnitude of some reference velocity. For simplicity of notation we omit the asterisks. Then, the reactor flow problem reads in dimensionless form as follows:

$$
\begin{aligned}
-\operatorname{div}\left(\frac{\varepsilon}{\operatorname{Re}} \nabla \mathbf{u}-\varepsilon \mathbf{u} \otimes \mathbf{u}\right)+\varepsilon \nabla p+\frac{\alpha}{\operatorname{Re}} \mathbf{u}+\beta \mathbf{u}|\mathbf{u}| & =\mathbf{f} \\
& \text { in } \Omega, \\
\operatorname{div}(\varepsilon \mathbf{u}) & =0 \\
& \text { in } \Omega, \\
\mathbf{u} & =\mathbf{g} \\
& \text { on } \Gamma,
\end{aligned}
$$

where

$$
\begin{aligned}
& \alpha(\mathbf{x})=150 \kappa^{2}(\mathbf{x}), \\
& \beta(\mathbf{x})=1.75 \kappa(\mathbf{x}),
\end{aligned}
$$

with

$$
\kappa(\mathbf{x})=\frac{1-\varepsilon(\mathbf{x})}{\varepsilon(\mathbf{x})},
$$

and the Reynolds number is defined by

$$
\operatorname{Re}=\frac{U_{0} d_{p}}{v} .
$$

The existence and uniqueness of the solution of a system for flow, temperature, and solute transport containing the nonlinear flow model (6) but with constant porosity and without the convective term have been established in [16]. We will extend this result for the flow component to the case (6) when the variable porosity depends on the location and the convective term is included. The recent existence results for the linear Brinkman problem with spatially varying $\alpha$ can be found in [17].

Remark 1. Equation (6) becomes a Navier-Stokes problem if $\varepsilon \equiv 1$.

Notation. Throughout the work we use the following notations for function spaces. For $m \in \mathbb{N}_{0}, p \geq 1$ and bounded subdomain $G \subset \Omega$; let $W^{m, p}(G)$ be the usual Sobolev space equipped with norm $\|\cdot\|_{m, p, G}$. If $p=2$, we denote the Sobolev space by $H^{m}(G)$ and use the standard abbreviations $\|\cdot\|_{m, G}$ and $|\cdot|_{m, G}$ for the norm and seminorm, respectively. We denote by $D(G)$ the space of $C^{\infty}(G)$ functions with compact support contained in $G$. Furthermore, $H_{0}^{m}(G)$ stands for the closure of $D(G)$ with respect to the norm $\|\cdot\|_{m, G}$. The counterparts spaces consisting of vector valued functions will be denoted by bold faced symbols like $\mathbf{H}^{m}(G):=\left[H^{m}(G)\right]^{n}$ or $\mathbf{D}(G):=[D(G)]^{n}$. The $L^{2}$ inner product over $G \subset \Omega$ and $\partial G \subset \partial \Omega$ will be denoted by $(\cdot, \cdot)_{G}$ and $\langle\cdot, \cdot\rangle_{\partial G}$, respectively. In the case $G=\Omega$ the domain index will be omitted. In the following we denote by $C$ the generic constant which is usually independent of the model parameters; otherwise dependence will be indicated.

\section{Existence and Uniqueness Results}

In the following the porosity $\varepsilon$ is assumed to belong to $W^{1,3}(\Omega) \cap L^{\infty}(\Omega)$. We start with the weak formulation of problem (6) and look for its solution in suitable Sobolev spaces that are adjusted to the modified momentum and mass balances in (6) and to the smoothness of the weighting function $\varepsilon$.

\subsection{Variational Formulation. Let}

$$
L_{0}^{2}(\Omega):=\left\{v \in L^{2}(\Omega):(v, 1)=0\right\}
$$

be the space consisting of $L^{2}$ functions with zero mean value. We define the spaces

$$
\begin{aligned}
\mathbf{X} & :=\mathbf{H}^{1}(\Omega), \\
\mathbf{X}_{0} & :=\mathbf{H}_{0}^{1}(\Omega), \\
\mathbf{Q} & :=L^{2}(\Omega), \\
M & :=L_{0}^{2}(\Omega), \\
\mathbf{V} & :=\mathbf{X}_{0} \times M .
\end{aligned}
$$


Let us introduce the following bilinear forms:

$$
\begin{aligned}
& a: \mathbf{X} \times \mathbf{X} \rightarrow \mathbb{R}, \quad a(\mathbf{u}, \mathbf{v})=\frac{1}{\operatorname{Re}}(\varepsilon \nabla \mathbf{u}, \nabla \mathbf{v}), \\
& b: \mathbf{X} \times Q \rightarrow \mathbb{R}, \quad b(\mathbf{u}, q)=(\operatorname{div}(\varepsilon \mathbf{u}), q), \\
& c: \mathbf{X} \times \mathbf{X} \longrightarrow \mathbb{R}, \quad c(\mathbf{u}, \mathbf{v})=\frac{1}{\operatorname{Re}}(\alpha \mathbf{u}, \mathbf{v}) .
\end{aligned}
$$

Furthermore, we define the semilinear form

$$
d: \mathbf{X} \times \mathbf{X} \times \mathbf{X} \longrightarrow \mathbb{R}, \quad d(\mathbf{w} ; \mathbf{u}, \mathbf{v})=(\beta|\mathbf{w}| \mathbf{u}, \mathbf{v}),
$$

and trilinear form

$$
n: \mathbf{X} \times \mathbf{X} \times \mathbf{X} \longrightarrow \mathbb{R}, \quad n(\mathbf{w}, \mathbf{u}, \mathbf{v})=((\varepsilon \mathbf{w} \cdot \nabla) \mathbf{u}, \mathbf{v}) .
$$

We set

$$
\begin{aligned}
A(\mathbf{w} ; \mathbf{u}, \mathbf{v}):= & a(\mathbf{u}, \mathbf{v})+c(\mathbf{u}, \mathbf{v})+n(\mathbf{w}, \mathbf{u}, \mathbf{v}) \\
& +d(\mathbf{w} ; \mathbf{u}, \mathbf{v})
\end{aligned}
$$

Multiplying momentum and mass balances in (6) by test functions $\mathbf{v} \in \mathbf{X}_{0}$ and $q \in M$, respectively, and integrating by parts implies the weak formulation

$$
\begin{aligned}
\text { Find } & (\mathbf{u}, p) \in \mathbf{X} \times M \text { with }\left.\mathbf{u}\right|_{\Gamma}=\mathbf{g} \\
\text { such that } \quad & A(\mathbf{u} ; \mathbf{u}, \mathbf{v})-b(\mathbf{v}, p)=(\mathbf{f}, \mathbf{v}), \\
& b(\mathbf{u}, q)=0 \\
& \forall \mathbf{v} \in \mathbf{X}_{0}, \forall q \in M .
\end{aligned}
$$

First, we recall the following result from [18].

Theorem 2. The mapping $u \mapsto \varepsilon u$ is an isomorphism from $H^{1}(\Omega)$ onto itself and from $H_{0}^{1}(\Omega)$ onto itself. It holds for all $u \in H^{1}(\Omega)$ that

$$
\begin{aligned}
& \|\varepsilon u\|_{1} \leq C\left\{\varepsilon_{1}+|\varepsilon|_{1,3}\right\}\|u\|_{1}, \\
& \left\|\frac{u}{\varepsilon}\right\|_{1} \leq C\left\{\varepsilon_{0}^{-1}+\varepsilon_{0}^{-2}|\varepsilon|_{1,3}\right\}\|u\|_{1} .
\end{aligned}
$$

In the following the closed subspace of $\mathbf{H}_{0}^{1}(\Omega)$ defined by

$$
\mathbf{W}=\left\{\mathbf{w} \in \mathbf{H}_{0}^{1}(\Omega): b(\mathbf{w}, q)=0 \forall q \in L_{0}^{2}(\Omega)\right\}
$$

will be employed. Next, we establish and prove some properties of trilinear form $n(\cdot, \cdot, \cdot)$ and nonlinear form $d(\cdot ; \cdot, \cdot)$.

Lemma 3. Let $\mathbf{u}, \mathbf{v} \in \mathbf{H}^{1}(\Omega)$ and $\mathbf{w} \in \mathbf{H}^{1}(\Omega)$ with $\operatorname{div}(\varepsilon \mathbf{w})=0$ and $\left.\mathbf{w} \cdot \mathbf{n}\right|_{\Gamma}=0$. Then we have

$$
n(\mathbf{w}, \mathbf{u}, \mathbf{v})=-n(\mathbf{w}, \mathbf{v}, \mathbf{u}) .
$$

Furthermore, the trilinear form $n(\cdot, \cdot, \cdot)$ and the nonlinear form $d(\cdot ; \cdot, \cdot)$ are continuous; that is,

$$
\begin{aligned}
& |n(\mathbf{u}, \mathbf{v}, \mathbf{w})| \leq C_{\varepsilon}\|\mathbf{u}\|_{1}\|\mathbf{v}\|_{1}\|\mathbf{w}\|_{1} \quad \forall \mathbf{u}, \mathbf{v}, \mathbf{w} \in \mathbf{H}^{1}(\Omega), \\
& |d(\mathbf{u}, \mathbf{v}, \mathbf{w})| \leq C_{\varepsilon}\|\mathbf{u}\|_{1}\|\mathbf{v}\|_{1}\|\mathbf{w}\|_{1} \quad \forall \mathbf{u}, \mathbf{v}, \mathbf{w} \in \mathbf{H}^{1}(\Omega),
\end{aligned}
$$

and for $\mathbf{u} \in \mathbf{W}$ and for a sequence $\mathbf{u}^{k} \in \mathbf{W}$ with $\lim _{k \rightarrow \infty} \| \mathbf{u}^{k}-$ $\mathbf{u} \|_{0}=0$, we have also

$$
\lim _{k \rightarrow \infty} n\left(\mathbf{u}^{k}, \mathbf{u}^{k}, \mathbf{v}\right)=n(\mathbf{u}, \mathbf{u}, \mathbf{v}) \quad \forall \mathbf{v} \in \mathbf{W} .
$$

Proof. We follow the proof of [19, Lemma 2.1, \$2, Chapter IV] and adapt it to the trilinear form

$$
n(\mathbf{w}, \mathbf{u}, \mathbf{v})=((\varepsilon \mathbf{w} \cdot \nabla) \mathbf{u}, \mathbf{v})=\sum_{i, j=1}^{n}\left(\varepsilon w_{j} \partial_{j} u_{i}, v_{i}\right),
$$

which has the weighting factor $\varepsilon$. Hereby, symbols with subscripts denote components of bold faced vectors, for example, $\mathbf{u}=\left(u_{i}\right)_{i=1, \ldots, n}$. Let $\mathbf{u} \in \mathbf{H}^{1}, \mathbf{v} \in \mathbf{D}(\Omega)$, and $\mathbf{w} \in \mathbf{W}$. Integrating by parts and employing density argument, we obtain immediately (19).

$$
\begin{aligned}
\sum_{i, j=1}^{n}\left(\varepsilon w_{j} \partial_{j} u_{i}, v_{i}\right)= & -\sum_{i, j=1}^{n}\left(\partial_{j}\left(\varepsilon w_{j} v_{i}\right), u_{i}\right) \\
& +\sum_{i, j=1}^{n}\left\langle\varepsilon w_{j} n_{j} u_{i}, v_{i}\right\rangle \\
= & -\sum_{i, j=1}^{n}\left(\varepsilon w_{j} \partial_{j} v_{i}, u_{i}\right) \\
& -(\operatorname{div}(\varepsilon \mathbf{w}) \mathbf{u}, \mathbf{v})+\langle(\varepsilon \mathbf{w} \cdot \mathbf{n}) \mathbf{u}, \mathbf{v}\rangle \\
= & -n(\mathbf{w}, \mathbf{v}, \mathbf{u}) .
\end{aligned}
$$

From Sobolev embedding $H^{1}(\Omega) \hookrightarrow L^{4}(\Omega)$ (see [20]) and Hölder inequality follows

$$
\begin{aligned}
\left|\left(\varepsilon w_{j} \partial_{j} u_{i}, v_{i}\right)\right| & \leq|\varepsilon|_{0, \infty}\left\|w_{j}\right\|_{0,4}\left\|\partial_{j} u_{i}\right\|_{0}\left\|v_{i}\right\|_{0,4} \\
& \leq C|\varepsilon|_{0, \infty}\left\|w_{j}\right\|_{1}\left|u_{i}\right|_{1}\left\|v_{i}\right\|_{1},
\end{aligned}
$$

and consequently the proof of (20) is completed. Since $\lim _{k \rightarrow \infty}\left\|u_{i}^{k} u_{j}^{k}-u_{i} u_{j}\right\|_{0,1}=0$ and $\varepsilon \partial_{j} v_{i} \in L^{\infty}(\Omega)$, the continuity estimate (20) implies

$$
\begin{aligned}
\lim _{k \rightarrow \infty} n\left(\mathbf{u}^{k}, \mathbf{u}^{k}, \mathbf{v}\right) & =-\lim _{k \rightarrow \infty} n\left(\mathbf{u}^{k}, \mathbf{v}, \mathbf{u}^{k}\right) \\
& =-\lim _{k \rightarrow \infty} \sum_{i, j=1}^{n}\left(\varepsilon u_{j}^{k} \partial_{j} v_{i}^{k}, u_{i}^{k}\right) \\
& =-\sum_{i, j=1}^{n}\left(\varepsilon u_{j} \partial_{j} v_{i}, u_{i}\right)=-n(\mathbf{u}, \mathbf{v}, \mathbf{u}) \\
& =n(\mathbf{u}, \mathbf{u}, \mathbf{v}) .
\end{aligned}
$$

The continuity of $d(\cdot ; \cdot, \cdot)$ follows from Hölder inequality and Sobolev embedding $H^{1}(\Omega) \hookrightarrow L^{4}(\Omega)$ (see [20]).

$$
\begin{aligned}
|d(\mathbf{u} ; \mathbf{v}, \mathbf{w})| & \leq|\beta|_{\infty}\|\mathbf{u}\|_{0,4}\|\mathbf{v}\|_{0,4}\|\mathbf{w}\|_{0} \\
& \leq C_{\varepsilon}\|\mathbf{u}\|_{1}\|\mathbf{v}\|_{1}\|\mathbf{w}\|_{1} .
\end{aligned}
$$


In the next stage we consider the difficulties caused by prescribing the inhomogeneous Dirichlet boundary condition. Analogous difficulties are already encountered in the analysis of Navier-Stokes problem. We will carry out the study of three-dimensional case. The extension in two dimensions can be constructed analogously. Since $\mathbf{g} \in \mathbf{H}^{1 / 2}(\Gamma)$, we can extend $\mathbf{g}$ inside of $\Omega$ in the form of

$$
\mathbf{g}=\varepsilon^{-1} \operatorname{curl} \mathbf{h},
$$

with some $\mathbf{h} \in \mathbf{H}^{2}(\Omega)$. The operator curl is defined then as

$$
\operatorname{curl} \mathbf{h}=\left(\partial_{2} h_{3}-\partial_{3} h_{2}, \partial_{3} h_{1}-\partial_{1} h_{3}, \partial_{1} h_{2}-\partial_{2} h_{1}\right) .
$$

We note that in the two-dimensional case the vector potential h $\in \mathbf{H}^{2}(\Omega)$ can be replaced by a scalar function $h \in$ $H^{2}(\Omega)$ and the operator curl is then redefined as curl $h=$ $\left(\partial_{2} h,-\partial_{1} h\right)$. Our aim is to adapt the extension of Hopf (see [21]) to our model. We recall that for any parameter $\mu>0$ there exists a scalar function $\varphi_{\mu} \in C^{2}(\bar{\Omega})$ such that

(i) $\varphi_{\mu}=1$ in some neighborhood of $\Gamma$ (depending on $\mu$ ),

(ii) $\varphi_{\mu}(\mathbf{x})=0$ if $d_{\Gamma}(\mathbf{x}) \geq 2 \exp \left(-\frac{1}{\mu}\right)$, where $d_{\Gamma}(\mathbf{x}):=\inf _{\mathbf{y} \in \Gamma}|\mathbf{x}-\mathbf{y}|$ denotes the distance of $\mathbf{x}$ to $\Gamma$,

(iii) $\left|\partial_{j} \varphi_{\mu}(\mathbf{x})\right| \leq \frac{\mu}{d_{\Gamma}(\mathbf{x})} \quad$ if $d_{\Gamma}(\mathbf{x})<2 \exp \left(-\frac{1}{\mu}\right), j=1, \ldots, n$.

For the construction of $\varphi_{\mu}$ see also [19, Lemma 2.4, $\$ 2$, Chapter IV].

Let us define

$$
\mathbf{g}_{\mu}:=\varepsilon^{-1} \operatorname{curl}\left(\varphi_{\mu} \mathbf{h}\right) .
$$

In the following lemma we establish bounds which are crucial for proving existence of velocity.

Lemma 4. The function $\mathbf{g}_{\mu}$ satisfies the following conditions:

$$
\begin{aligned}
\operatorname{div}\left(\varepsilon \mathbf{g}_{\mu}\right) & =0, \\
\left.\mathbf{g}_{\mu}\right|_{\Gamma} & =\mathbf{g},
\end{aligned}
$$

$$
\forall \mu>0,
$$

and for any $\delta>0$ there exists sufficiently small $\mu>0$ such that

$$
\begin{gathered}
\left|d\left(\mathbf{u}+\mathbf{g}_{\mu} ; \mathbf{g}_{\mu}, \mathbf{u}\right)\right| \leq \delta\|\beta\|_{0, \infty}|\mathbf{u}|_{1}\left(|\mathbf{u}|_{1}+\left\|\mathbf{g}_{\mu}\right\|_{0}\right) \\
\forall \mathbf{u} \in \mathbf{X}_{0}, \\
\left|n\left(\mathbf{u}, \mathbf{g}_{\mu}, \mathbf{u}\right)\right| \leq \delta|\mathbf{u}|_{1}^{2} \quad \forall \mathbf{u} \in \mathbf{W} .
\end{gathered}
$$

Proof. The relations in (31) are obvious. We follow [16] in order to show (32). Since $\mathbf{h} \in \mathbf{H}^{2}(\Omega)$ Sobolev's embedding theorem implies $\mathbf{h} \in \mathbf{L}^{\infty}(\Omega)$, so we get according to the properties of $\varphi_{\mu}$ in (Ex) the following bound:

$$
\begin{aligned}
\left|\mathbf{g}_{\mu}\right| & \leq C \varepsilon_{0}^{-1}\left\{|\nabla \mathbf{h}|+\frac{\mu}{d_{\Gamma}(\mathbf{x})}|\mathbf{h}|\right\} \\
& \leq C\left\{\frac{\mu}{d_{\Gamma}(\mathbf{x})}+|\nabla \mathbf{h}|\right\} .
\end{aligned}
$$

Defining

$$
\Omega_{\mu}:=\left\{\mathbf{x} \in \Omega: d_{\Gamma}(\mathbf{x})<2 \exp \left(-\frac{1}{\mu}\right)\right\},
$$

we obtain from Cauchy-Schwarz and triangle inequalities

$$
\begin{aligned}
& \left|\left(\beta\left|\mathbf{u}+\mathbf{g}_{\mu}\right|, \mathbf{g}_{\mu} \cdot \mathbf{u}\right)\right| \leq\|\beta\|_{0, \infty}\|\mathbf{u}\|_{0}\left\|\mathbf{u} \cdot \mathbf{g}_{\mu}\right\|_{0, \Omega_{\mu}} \\
& +\|\beta\|_{0, \infty}\left\|\mathbf{g}_{\mu}\right\|_{0}\left\|\mathbf{u} \cdot \mathbf{g}_{\mu}\right\|_{0, \Omega_{\mu}}, \\
& \left\|\mathbf{u} \cdot \mathbf{g}_{\mu}\right\|_{0, \Omega_{\mu}}^{2} \leq \int_{\Omega_{\mu}}|\mathbf{u}|^{2}\left|\mathbf{g}_{\mu}\right|^{2} d \mathbf{x} \leq C \int_{\Omega_{\mu}}|\mathbf{u}|^{2} \\
& \cdot\left\{\left(\frac{\mu}{d_{\Gamma}(\mathbf{x})}\right)^{2}+\frac{2 \mu}{d_{\Gamma}(\mathbf{x})|\nabla \mathbf{h}|}+|\nabla \mathbf{h}|^{2}\right\} d \mathbf{x} \\
& \leq C\left\{\mu^{2}\left\|\frac{\mathbf{u}}{d_{\Gamma}}\right\|_{0, \Omega_{\mu}}^{2}+2 \mu\left\|\frac{\mathbf{u}}{d_{\Gamma}}\right\|_{0, \Omega_{\mu}}\|\mathbf{u}\|_{0,4, \Omega_{\mu}}\right. \\
& \left.\cdot\||\nabla \mathbf{h}|\|_{0,4, \Omega_{\mu}}+\|\mathbf{u}\|_{0,4, \Omega_{\mu}}^{2}\||\nabla \mathbf{h}|\|_{0,4, \Omega_{\mu}}^{2}\right\} \\
& \leq C\left\{\mu\left\|\frac{\mathbf{u}}{d_{\Gamma}}\right\|_{0, \Omega_{\mu}}+\|\mathbf{u}\|_{0,4}\||\nabla \mathbf{h}|\|_{0,4, \Omega_{\mu}}\right\},
\end{aligned}
$$

and consequently

$$
\left\|\mathbf{u} \cdot \mathbf{g}_{\mu}\right\|_{0, \Omega_{\mu}} \leq C\left\{\mu\left\|\frac{\mathbf{u}}{d_{\Gamma}}\right\|_{0, \Omega_{\mu}}+\|\mathbf{u}\|_{0,4}\||\nabla \mathbf{h}|\|_{0,4, \Omega_{\mu}}\right\} .
$$

Applying Hardy inequality (see [20])

$$
\left\|\frac{v}{d_{\Gamma}}\right\|_{0} \leq C|v|_{1} \quad \forall v \in H_{0}^{1}(\Omega),
$$

and using Sobolev embedding $H^{1}(\Omega) \hookrightarrow L^{4}(\Omega)$, estimate (38) becomes

$$
\left\|\mathbf{u} \cdot \mathbf{g}_{\mu}\right\|_{0, \Omega_{\mu}} \leq C \lambda(\mu)\|\mathbf{u}\|_{1},
$$


where

$$
\lambda(\mu):=\max \left\{\mu,\||\nabla \mathbf{h}|\|_{0,4, \Omega_{\mu}}\right\} .
$$

From (36) and (40), Poincare inequality, and the fact that $\lim _{\mu \rightarrow 0} \lambda(\mu)=0$ we conclude that for any $\delta>0$ we can choose sufficiently small $\mu>0$ such that

$$
\left|\left(\beta\left|\mathbf{u}+\mathbf{g}_{\mu}\right| \mathbf{g}_{\mu}, \mathbf{u}\right)\right| \leq \delta\|\beta\|_{0, \infty}|\mathbf{u}|_{1}\left(|\mathbf{u}|_{1}+\left\|\mathbf{g}_{\mu}\right\|_{0}\right),
$$

holds. Therefore the proof of estimate (32) is completed. Now, we take a look at the trilinear convective term

$$
\begin{aligned}
n\left(\mathbf{u}, \mathbf{g}_{\mu}, \mathbf{u}\right)= & \left((\varepsilon \mathbf{u} \cdot \nabla) \mathbf{g}_{\mu}, \mathbf{u}\right)_{\Omega_{\mu}} \\
= & \left((\varepsilon \mathbf{u} \cdot \nabla)\left\{\varepsilon^{-1} \operatorname{curl}\left(\varphi_{\mu} \mathbf{h}\right)\right\}, \mathbf{u}\right)_{\Omega_{\mu}} \\
= & \left((\mathbf{u} \cdot \nabla)\left\{\operatorname{curl}\left(\varphi_{\mu} \mathbf{h}\right)\right\}, \mathbf{u}\right)_{\Omega_{\mu}} \\
& -\left((\mathbf{u} \cdot \nabla \varepsilon) \mathbf{g}_{\mu}, \mathbf{u}\right)_{\Omega_{\mu}} .
\end{aligned}
$$

The first term of above difference becomes small due to [19, Lemma 2.3, \$2, Chapter IV], and it satisfies

$$
\begin{aligned}
& \left|\left((\mathbf{u} \cdot \nabla)\left\{\operatorname{curl}\left(\varphi_{\mu} \mathbf{h}\right)\right\}, \mathbf{u}\right)_{\Omega_{\mu}}\right|=\left|\left((\mathbf{u} \cdot \nabla)\left(\varepsilon \mathbf{g}_{\mu}\right), \mathbf{u}\right)_{\Omega_{\mu}}\right| \\
& \quad \leq \delta|\mathbf{u}|_{1}^{2},
\end{aligned}
$$

as long as $\mu>0$ is chosen sufficiently small. Using Hölder inequality, Sobolev embedding $H^{1}(\Omega) \hookrightarrow L^{6}(\Omega)$ yields

$$
\left|\left((\mathbf{u} \cdot \nabla \varepsilon) \mathbf{g}_{\mu}, \mathbf{u}\right)_{\Omega_{\mu}}\right| \leq C\|\varepsilon\|_{1,3}\left\|\mathbf{g}_{\mu} \cdot \mathbf{u}\right\|_{0}\|\mathbf{u}\|_{1},
$$

which together with (40) implies for sufficiently small $\mu>0$ the bound

$$
\left|\left((\mathbf{u} \cdot \nabla \varepsilon) \mathbf{g}_{\mu}, \mathbf{u}\right)_{\Omega_{\mu}}\right| \leq \delta|\mathbf{u}|_{1}^{2} .
$$

From (44) and (46) follows the desired estimate (33).

While the general framework for linear and nonsymmetric saddle point problems can be found in [18], our problem requires more attention due to its nonlinear character. Setting $\mathbf{w}:=\mathbf{u}-\mathbf{g}_{\mu}$, the weak formulation (16) is equivalent to the following problem:

$$
\text { Find }(\mathbf{w}, p) \in \mathbf{V}
$$

$$
\begin{aligned}
& \text { such that } A\left(\mathbf{w}+\mathbf{g}_{\mu} ; \mathbf{w}+\mathbf{g}_{\mu}, \mathbf{v}\right)-b(\mathbf{v}, p) \\
& +b\left(\mathbf{w}+\mathbf{g}_{\mu}, q\right)=(\mathbf{f}, \mathbf{v}) \quad \forall(\mathbf{v}, q) \in \mathbf{V} .
\end{aligned}
$$

Let us define the nonlinear mapping $G: \mathbf{W} \rightarrow \mathbf{W}$ with

$$
\begin{aligned}
{[G(\mathbf{w}), \mathbf{v}]:=} & a\left(\mathbf{w}+\mathbf{g}_{\mu}, \mathbf{v}\right)+c\left(\mathbf{w}+\mathbf{g}_{\mu}, \mathbf{v}\right)-(\mathbf{f}, \mathbf{v}) \\
& +n\left(\mathbf{w}+\mathbf{g}_{\mu}, \mathbf{w}+\mathbf{g}_{\mu}, \mathbf{v}\right) \\
& +d\left(\mathbf{w}+\mathbf{g}_{\mu} ; \mathbf{w}+\mathbf{g}_{\mu}, \mathbf{v}\right)
\end{aligned}
$$

where $[\cdot, \cdot]$ defines the inner product in $\mathbf{W}$ via $[u, v]:=$ $(\nabla u, \nabla v)$. Then, the variational problem (47) reads in the space $\mathbf{W}$ as follows.

Find $\mathbf{w} \in \mathbf{W}$ such that

$$
[G(\mathbf{w}), \mathbf{v}]=0 \quad \forall \mathbf{v} \in \mathbf{W} .
$$

2.2. Solvability of Nonlinear Saddle Point Problem. We start our study of the nonlinear operator problem (49) with the following lemma.

Lemma 5. The mapping $G$ defined in (48) is continuous and there exists $r>0$ such that

$$
[G(\mathbf{u}), \mathbf{u}]>0 \quad \forall \mathbf{u} \in \mathbf{W} \text { with }|\mathbf{u}|_{1}=r .
$$

Proof. Let $\left(\mathbf{u}^{k}\right)_{k \in \mathbb{N}}$ be a sequence in $\mathbf{W}$ with $\lim _{k \rightarrow \infty} \| \mathbf{u}^{k}-$ $\mathbf{u} \|_{1}=0$. Then, applying Cauchy-Schwarz inequality and (33), we obtain for any $\mathbf{v} \in \mathbf{W}$

$$
\begin{aligned}
& \left|\left[G\left(\mathbf{u}^{k}\right)-G(\mathbf{u}), \mathbf{v}\right]\right| \\
& \leq \\
& \quad \frac{1}{\operatorname{Re}}\left|\left(\varepsilon \nabla\left(\mathbf{u}^{k}-\mathbf{u}\right), \nabla \mathbf{v}\right)\right|+\frac{1}{\operatorname{Re}}\left|\left(\alpha\left(\mathbf{u}^{k}-\mathbf{u}\right), \mathbf{v}\right)\right| \\
& \quad+\left|\left(\beta\left|\mathbf{u}^{k}+\mathbf{g}_{\mu}\right|\left(\mathbf{u}^{k}-\mathbf{u}\right), \mathbf{v}\right)\right| \\
& \quad+\left|\left(\beta\left(\left|\mathbf{u}^{k}+\mathbf{g}_{\mu}\right|-\left|\mathbf{u}+\mathbf{g}_{\mu}\right|\right)\left(\mathbf{u}+\mathbf{g}_{\mu}\right), \mathbf{v}\right)\right| \\
& \quad+\left|n\left(\mathbf{u}^{k}, \mathbf{u}^{k}, \mathbf{v}\right)-n(\mathbf{u}, \mathbf{u}, \mathbf{v})\right|+\left|n\left(\mathbf{u}^{k}-\mathbf{u}, \mathbf{g}_{\mu}, \mathbf{v}\right)\right| \\
& \quad+\left|n\left(\mathbf{g}_{\mu}, \mathbf{u}^{k}-\mathbf{u}, \mathbf{v}\right)\right| \\
& \leq \frac{\varepsilon_{1}}{\operatorname{Re}}\left|\mathbf{u}^{k}-\mathbf{u}\right|_{1}|\mathbf{v}|_{1}+\frac{1}{\operatorname{Re}}\|\alpha\|_{0, \infty}\left\|\mathbf{u}^{k}-\mathbf{u}\right\|_{0}\|\mathbf{v}\|_{0} \\
& \quad+\|\beta\|_{0, \infty}\left\|\mathbf{u}^{k}+\mathbf{g}_{\mu}\right\|_{0,4}\left\|\mathbf{u}^{k}-\mathbf{u}\right\|_{0}\|\mathbf{v}\|_{0,4} \\
& \quad+\|\beta\|_{0, \infty}\left\|\mathbf{u}+\mathbf{g}_{\mu}\right\|_{0,4}\left\|\mathbf{u}^{k}-\mathbf{u}\right\|_{0}\|\mathbf{v}\|_{0,4} \\
& \quad+\left|n\left(\mathbf{u}^{k}, \mathbf{u}^{k}, \mathbf{v}\right)-n(\mathbf{u}, \mathbf{u}, \mathbf{v})\right| \\
& \quad+C\left\|\mathbf{u}^{k}-\mathbf{u}\right\|_{1}\left\|\mathbf{g}_{\mu}\right\|_{1}\|\mathbf{v}\|_{1} .
\end{aligned}
$$

The boundedness of $\mathbf{u}^{k}$ in $\mathbf{W},(22)$, the Poincare inequality, and the above inequality imply that

$$
\left|\left[G\left(\mathbf{u}^{k}\right)-G(\mathbf{u}), \mathbf{v}\right]\right| \longrightarrow 0 \quad \text { as } k \longrightarrow \infty \forall \mathbf{v} \in \mathbf{W} .
$$

Thus, employing

$$
\left|G\left(\mathbf{u}^{k}\right)-G(\mathbf{u})\right|_{1}=\sup _{\substack{\mathbf{v} \in \mathbf{W} \\ \mathbf{v} \neq \mathbf{0}}} \frac{\left[G\left(\mathbf{u}^{k}\right)-G(\mathbf{u}), \mathbf{v}\right]}{|\mathbf{v}|_{1}},
$$


we state that $G$ is continuous. Now, we note that for any $\mathbf{u} \in \mathbf{W}$ we have

$$
\begin{aligned}
{[G(\mathbf{u}), \mathbf{u}]=} & \frac{1}{\operatorname{Re}}\left(\varepsilon \nabla\left(\mathbf{u}+\mathbf{g}_{\mu}\right), \nabla \mathbf{u}\right) \\
& +\frac{1}{\operatorname{Re}}\left(\alpha\left(\mathbf{u}+\mathbf{g}_{\mu}\right), \mathbf{u}\right) \\
& +\left(\beta\left|\mathbf{u}+\mathbf{g}_{\mu}\right|\left(\mathbf{u}+\mathbf{g}_{\mu}\right), \mathbf{u}\right) \\
& +n\left(\mathbf{u}+\mathbf{g}_{\mu}, \mathbf{u}+\mathbf{g}_{\mu}, \mathbf{u}\right)-(\mathbf{f}, \mathbf{u}) \\
\geq & \frac{\varepsilon_{0}}{\operatorname{Re}}|\mathbf{u}|_{1}^{2}-\frac{\varepsilon_{1}}{\operatorname{Re}}\left|\left(\nabla \mathbf{g}_{\mu}, \nabla \mathbf{u}\right)\right|+\frac{1}{\operatorname{Re}}(\alpha \mathbf{u}, \mathbf{u}) \\
& -\frac{1}{\operatorname{Re}}\left|\left(\alpha \mathbf{g}_{\mu}, \mathbf{u}\right)\right|+\left(\beta\left|\mathbf{u}+\mathbf{g}_{\mu}\right|,|\mathbf{u}|^{2}\right) \\
& -\left|\left(\beta\left|\mathbf{u}+\mathbf{g}_{\mu}\right| \mathbf{g}_{\mu}, \mathbf{u}\right)\right|+n\left(\mathbf{u}, \mathbf{g}_{\mu}, \mathbf{u}\right) \\
& +n\left(\mathbf{g}_{\mu}, \mathbf{g}_{\mu}, \mathbf{u}\right)-\|\mathbf{f}\|_{0}\|\mathbf{u}\|_{0} \\
\geq & \frac{\varepsilon_{0}}{\operatorname{Re}}|\mathbf{u}|_{1}^{2}-\left.\frac{\varepsilon_{1}}{\operatorname{Re}}\left|\mathbf{g}_{\mu}\right|\right|_{1}|\mathbf{u}|_{1} \\
& -\frac{1}{\operatorname{Re}}\|\alpha\|_{0, \infty}\left\|\mathbf{g}_{\mu}\right\|_{0}\|\mathbf{u}\|_{0} \\
& -\left|\left(\beta\left|\mathbf{u}+\mathbf{g}_{\mu}\right| \mathbf{g}_{\mu}, \mathbf{u}\right)\right|-\left|n\left(\mathbf{u}, \mathbf{g}_{\mu}, \mathbf{u}\right)\right| \\
& -\left\|\mathbf{g}_{\mu}\right\|_{1}^{2}\|\mathbf{u}\|_{1}-\|\mathbf{f}\|_{0}\|\mathbf{u}\|_{0} . \\
& \\
&
\end{aligned}
$$

From the Poincaré inequality, we infer the estimate

$$
\|v\|_{1} \leq C|v|_{1} \quad \forall v \in H_{0}^{1}(\Omega)
$$

which together with (32), (33), and (54) results in

$$
\begin{aligned}
& {[G(\mathbf{u}), \mathbf{u}] \geq\left\{\frac{\varepsilon_{0}}{\operatorname{Re}}-\delta\left(1+\|\beta\|_{0, \infty}\right)\right\}|\mathbf{u}|_{1}^{2}-\left\{\frac{\varepsilon_{1}}{\operatorname{Re}}\left|\mathbf{g}_{\mu}\right|_{1}\right.} \\
& +C_{1} \frac{1}{\operatorname{Re}}\|\alpha\|_{0, \infty}\left\|\mathbf{g}_{\mu}\right\|_{0}+\delta\|\beta\|_{0, \infty}\left\|\mathbf{g}_{\mu}\right\|_{0}+C_{2}\left\|\mathbf{g}_{\mu}\right\|_{1}^{2} \\
& \left.\quad+C_{3}\|\mathbf{f}\|_{0}\right\}|\mathbf{u}|_{1} .
\end{aligned}
$$

Choosing $\delta$ such that

$$
0<\delta<\delta_{0}:=\frac{\varepsilon_{0}}{\operatorname{Re}}\left(1+\|\beta\|_{0, \infty}\right)^{-1},
$$

and $r>r_{0}$ with

$$
r_{0}:=\frac{\left(\varepsilon_{1} / \mathrm{Re}\right)\left|\mathbf{g}_{\mu}\right|_{1}+(1 / \mathrm{Re}) C_{1}\|\alpha\|_{0, \infty}\left\|\mathbf{g}_{\mu}\right\|_{0}+\delta\|\beta\|_{0, \infty}\left\|\mathbf{g}_{\mu}\right\|_{0}+C_{2}\left\|\mathbf{g}_{\mu}\right\|_{1}^{2}+C_{3}\|\mathbf{f}\|_{0}}{\varepsilon_{0} / \operatorname{Re}-\delta\left(1+\|\beta\|_{0, \infty}\right)},
$$

leads to the desired assertion (50). proof.

The following lemma plays a key role in the existence

Lemma 6. Let $Y$ be finite dimensional Hilbert space with inner product $[\cdot, \cdot]$ inducing a norm $\|\cdot\|$ and $T: Y \rightarrow Y$ be a continuous mapping such that

$$
[T(x), x]>0 \text { for }\|x\|=r_{0}>0 .
$$

Then there exists $x \in Y$, with $\|x\| \leq r_{0}$, such that

$$
T(x)=0 .
$$

Proof. See [22].

Now we are able to prove the main result concerning existence of velocity.

Theorem 7. The problem (49) has at least one solution $\mathbf{u} \in \mathbf{W}$.

Proof. We construct the approximate sequence of Galerkin solutions. Since the space $\mathbf{W}$ is separable, there exists a sequence of linearly independent elements $\left(\mathbf{w}^{i}\right)_{i \in \mathbb{N}} \subset \mathbf{W}$. Let $\mathbf{X}_{m}$ be the finite dimensional subspace of $\mathbf{W}$ with

$$
\mathbf{X}_{m}:=\operatorname{span}\left\{\mathbf{w}^{i}, i=1, \ldots, m\right\}
$$

and endowed with the scalar product of $\mathbf{W}$. Let $\mathbf{u}^{m}=$ $\sum_{j=1}^{m} a_{j} \mathbf{w}^{j}, a_{j} \in \mathbb{R}$, be a Galerkin solution of (49) defined by

$$
\left[G\left(\mathbf{u}^{m}\right), \mathbf{w}^{j}\right]=0, \quad \forall j=1, \ldots, m
$$

From Lemmas 5 and 6 we conclude that

$$
\left[G\left(\mathbf{u}^{m}\right), \mathbf{w}\right]=0 \quad \forall \mathbf{w} \in \mathbf{X}_{m}
$$

has a solution $\mathbf{u}^{m} \in \mathbf{X}_{m}$. The unknown coefficients $a_{j}$ can be obtained from the algebraic system (62). On the other 
hand, multiplying (62) by $a_{j}$ and adding the equations for $j=1, \ldots, m$ we have

$$
\begin{aligned}
0= & {\left[G\left(\mathbf{u}^{m}\right), \mathbf{u}^{m}\right] \geq\left\{\frac{1}{\operatorname{Re}}-\delta\left(1+\|\beta\|_{0, \infty}\right)\right\}\left|\mathbf{u}^{m}\right|_{1}^{2} } \\
& -\left\{\frac{1}{\operatorname{Re}}\left|\mathbf{g}_{\mu}\right|_{1}+C_{1} \frac{1}{\operatorname{Re}}\|\alpha\|_{0, \infty}\left\|\mathbf{g}_{\mu}\right\|_{0}\right. \\
& \left.+\delta\|\beta\|_{0, \infty}\left\|\mathbf{g}_{\mu}\right\|_{0}+C_{2}\left\|\mathbf{g}_{\mu}\right\|_{1}^{2}+C_{3}\|\mathbf{f}\|_{0}\right\}\left|\mathbf{u}^{m}\right|_{1} .
\end{aligned}
$$

This gives together with (58) the uniform boundedness in $\mathbf{W}$

$$
\left|\mathbf{u}^{m}\right|_{1} \leq r_{0}
$$

therefore there exists $\mathbf{u} \in \mathbf{W}$ and a subsequence $m_{k} \rightarrow \infty$ (we write for the convenience $m$ instead of $m_{k}$ ) such that

$$
\mathbf{u}^{m} \rightarrow \mathbf{u} \text { in } \mathbf{W} \text {. }
$$

Furthermore, the compactness of embedding $H^{1}(\Omega) \hookrightarrow$ $L^{4}(\Omega)$ implies

$$
\mathbf{u}^{m} \longrightarrow \mathbf{u} \quad \text { in } \mathbf{L}^{4}(\Omega) .
$$

Taking the limit in (63) with $m \rightarrow \infty$ we get

$$
[G(\mathbf{u}), \mathbf{w}]=0 \quad \forall \mathbf{w} \in \mathbf{X}_{m} .
$$

Finally, we apply the continuity argument and state that (68) is preserved for any $\mathbf{w} \in \mathbf{W}$; therefore $\mathbf{u}$ is the solution of (49).

For the reconstruction of the pressure we need inf-suptheorem.

Theorem 8. Assume that the bilinear form $b(\cdot, \cdot)$ satisfies the inf-sup condition

$$
\inf _{q \in M} \sup _{\mathbf{v} \in \mathbf{X}_{0}} \frac{b(\mathbf{v}, q)}{|\mathbf{v}|_{1}\|q\|_{0}} \geq \gamma>0 .
$$

Then, for each solution $\mathbf{u}$ of the nonlinear problem (49) there exists a unique pressure $p \in M$ such that the pair $(\mathbf{u}, p) \in \mathbf{V}$ is a solution of the homogeneous problem (47).

Proof. See [19, Theorem 1.4, \$1, Chapter IV].

We end up this subsection by proving the existence of the pressure.

Theorem 9. Let $\mathbf{w}$ be solution of problem (49). Then, there exists unique pressure $p \in M$.

Proof. We verify the inf-sup condition (69) of Theorem 8 by employing the isomorphism of Theorem 2. From [19, Corollary 2.4, Section 2, Chapter I] follows that for any $q$ in $L_{0}^{2}(\Omega)$ there exists $\mathbf{v}$ in $\mathbf{H}_{0}^{1}(\Omega)$ such that

$$
(\operatorname{div} \mathbf{v}, q) \geq \gamma^{*}\|\mathbf{v}\|_{1}\|q\|_{0},
$$

with a positive constant $\gamma^{*}$. Setting $\mathbf{u}=\mathbf{v} / \varepsilon$ and applying the isomorphism in Theorem 2, we obtain the estimate

$$
b(\mathbf{u}, q)=(\operatorname{div} \mathbf{v}, q) \geq \gamma^{*}\|\mathbf{v}\|_{1}\|q\|_{0} \geq \gamma_{\varepsilon}\|\mathbf{u}\|_{1}\|q\|_{0},
$$

where $\gamma_{\varepsilon}=\gamma^{*} / C\left\{\varepsilon_{0}^{-1}+\varepsilon_{0}^{-2}|\varepsilon|_{1,3}\right\}$. From the above estimate we conclude the inf-sup condition (69).
2.3. Uniqueness of Weak Solution. We exploit a priori estimates in order to prove uniqueness of weak velocity and pressure.

Theorem 10. If $\left\|\mathbf{g}_{\mu}\right\|_{1},\|\mathbf{f}\|_{-1}:=\sup _{\mathbf{0} \neq \mathbf{v} \in \mathbf{H}^{1}(\Omega)}\left((\mathbf{f}, \mathbf{v}) /\|\mathbf{v}\|_{1}\right)$ are sufficiently small; then the solution of (49) is unique.

Proof. Assume that $\left(\mathbf{u}_{1}, p_{1}\right)$ and $\left(\mathbf{u}_{2}, p_{2}\right)$ are two different solutions of (47). From (19) in Lemma 3 we obtain $n(\mathbf{w}, \mathbf{u}, \mathbf{u})=0 \forall \mathbf{w}, \mathbf{u} \in \mathbf{W}$. Then, we obtain

$$
\begin{aligned}
& 0=\left[G\left(\mathbf{u}_{1}\right)-G\left(\mathbf{u}_{2}\right), \mathbf{u}_{1}-\mathbf{u}_{2}\right]=a\left(\mathbf{u}_{1}-\mathbf{u}_{2}, \mathbf{u}_{1}-\mathbf{u}_{2}\right) \\
& +c\left(\mathbf{u}_{1}-\mathbf{u}_{2}, \mathbf{u}_{1}-\mathbf{u}_{2}\right)-\left(\mathbf{f}, \mathbf{u}_{1}-\mathbf{u}_{2}\right) \\
& +n\left(\mathbf{u}_{1}+\mathbf{g}_{\mu}, \mathbf{u}_{1}+\mathbf{g}_{\mu}, \mathbf{u}_{1}-\mathbf{u}_{2}\right) \\
& -n\left(\mathbf{u}_{2}+\mathbf{g}_{\mu}, \mathbf{u}_{2}+\mathbf{g}_{\mu}, \mathbf{u}_{1}-\mathbf{u}_{2}\right) \\
& +\left(\beta\left|\mathbf{u}_{1}+\mathbf{g}_{\mu}\right|\left(\mathbf{u}_{1}+\mathbf{g}_{\mu}\right), \mathbf{u}_{1}-\mathbf{u}_{2}\right) \\
& -\left(\beta\left|\mathbf{u}_{2}+\mathbf{g}_{\mu}\right|\left(\mathbf{u}_{2}+\mathbf{g}_{\mu}\right), \mathbf{u}_{1}-\mathbf{u}_{2}\right) \\
& \geq \frac{\varepsilon_{0}}{\operatorname{Re}}\left|\mathbf{u}_{1}-\mathbf{u}_{2}\right|_{1}^{2}-\|\mathbf{f}\|_{-1}\left\|\mathbf{u}_{1}-\mathbf{u}_{2}\right\|_{1} \\
& +n\left(\mathbf{u}_{1}-\mathbf{u}_{2}, \mathbf{u}_{2}+\mathbf{g}_{\mu}, \mathbf{u}_{1}-\mathbf{u}_{2}\right) \\
& +\left(\beta\left|\mathbf{u}_{1}+\mathbf{g}_{\mu}\right|\left(\mathbf{u}_{1}-\mathbf{u}_{2}\right), \mathbf{u}_{1}-\mathbf{u}_{2}\right) \\
& +\left(\beta\left(\left|\mathbf{u}_{1}+\mathbf{g}_{\mu}\right|-\left|\mathbf{u}_{2}+\mathbf{g}_{\mu}\right|\right)\left(\mathbf{u}_{2}+\mathbf{g}_{\mu}\right), \mathbf{u}_{1}-\mathbf{u}_{2}\right) \\
& \geq \frac{\varepsilon_{0}}{\operatorname{Re}}\left|\mathbf{u}_{1}-\mathbf{u}_{2}\right|_{1}^{2}-\|\mathbf{f}\|_{-1}\left\|\mathbf{u}_{1}-\mathbf{u}_{2}\right\|_{1} \\
& -\left|n\left(\mathbf{u}_{1}-\mathbf{u}_{2}, \mathbf{u}_{2}, \mathbf{u}_{1}-\mathbf{u}_{2}\right)\right| \\
& -\left|n\left(\mathbf{u}_{1}-\mathbf{u}_{2}, \mathbf{g}_{\mu}, \mathbf{u}_{1}-\mathbf{u}_{2}\right)\right|-\|\beta\|_{0, \infty} \\
& \cdot\left|\left(\left|\mathbf{u}_{1}+\mathbf{g}_{\mu}\right| \cdot\left|\mathbf{u}_{1}-\mathbf{u}_{2}\right|,\left|\mathbf{u}_{1}-\mathbf{u}_{2}\right|\right)\right|-\|\beta\|_{0, \infty} \\
& \cdot\left|\left(|| \mathbf{u}_{1}+\mathbf{g}_{\mu}|-| \mathbf{u}_{2}+\mathbf{g}_{\mu}|| \cdot\left|\mathbf{u}_{2}+\mathbf{g}_{\mu}\right|,\left|\mathbf{u}_{1}-\mathbf{u}_{2}\right|\right)\right| \cdot
\end{aligned}
$$

From Cauchy-Schwarz inequality and Sobolev embedding $H^{1}(\Omega) \hookrightarrow L^{4}(\Omega)$ we deduce

$$
\begin{aligned}
& \left|\left(\left|\mathbf{u}_{1}+\mathbf{g}_{\mu}\right| \cdot\left|\mathbf{u}_{1}-\mathbf{u}_{2}\right|,\left|\mathbf{u}_{1}-\mathbf{u}_{2}\right|\right)\right| \\
& \quad \leq C\left\{\left\|\mathbf{u}_{1}\right\|_{0}+\left\|\mathbf{g}_{\mu}\right\|_{0}\right\}\left\|\mathbf{u}_{1}-\mathbf{u}_{2}\right\|_{1}^{2}, \\
& \left|\left(\| \mathbf{u}_{1}+\mathbf{g}_{\mu}|-| \mathbf{u}_{2}+\mathbf{g}_{\mu}|| \cdot\left|\mathbf{u}_{2}+\mathbf{g}_{\mu}\right|,\left|\mathbf{u}_{1}-\mathbf{u}_{2}\right|\right)\right| \\
& \quad \leq C\left\{\left\|\mathbf{u}_{2}\right\|_{0}+\left\|\mathbf{g}_{\mu}\right\|_{0}\right\}\left\|\mathbf{u}_{1}-\mathbf{u}_{2}\right\|_{1}^{2},
\end{aligned}
$$

and according to (20) we have

$$
\left|n\left(\mathbf{u}_{1}-\mathbf{u}_{2}, \mathbf{u}_{2}, \mathbf{u}_{1}-\mathbf{u}_{2}\right)\right| \leq C\left\|\mathbf{u}_{2}\right\|_{1}\left\|\mathbf{u}_{1}-\mathbf{u}_{2}\right\|_{1}^{2},
$$

and by (31) we can find $\mu$ such that

$$
\left|n\left(\mathbf{u}_{1}-\mathbf{u}_{2}, \mathbf{g}_{\mu}, \mathbf{u}_{1}-\mathbf{u}_{2}\right)\right| \leq \frac{\varepsilon_{0}}{4 \operatorname{Re}}\left\|\mathbf{u}_{1}-\mathbf{u}_{2}\right\|_{1}^{2} .
$$


Now, we find upper bounds for $\mathbf{u}_{1}$ and $\mathbf{u}_{2}$. Testing (47) with $\mathbf{u}$ results in

$$
\begin{aligned}
\frac{\varepsilon_{0}}{\operatorname{Re}}\|\mathbf{u}\|_{1}^{2} \leq & \|\mathbf{f}\|_{-1}\|\mathbf{u}\|_{1}+\frac{\varepsilon_{0}}{\operatorname{Re}}\left\|\mathbf{g}_{\mu}\right\|_{1}\|\mathbf{u}\|_{1}+C\left\|\mathbf{g}_{\mu}\right\|_{0}\|\mathbf{u}\|_{0} \\
& +C\left\|\mathbf{g}_{\mu}\right\|_{1}^{2}\|\mathbf{u}\|_{1}+C\|\beta\|_{0, \infty}\left\|\mathbf{g}_{\mu}\right\|\left\|_{0}\right\| \mathbf{u} \|_{1}^{2} \\
& +C\|\beta\|_{0, \infty}\left\|\mathbf{g}_{\mu}\right\|_{0,4}^{2}\|\mathbf{u}\|_{1} .
\end{aligned}
$$

From Sobolev embedding $H^{1}(\Omega) \hookrightarrow L^{4}(\Omega)$ we deduce for sufficiently small $\left\|\mathbf{g}_{\mu}\right\|_{1}$

$$
\begin{aligned}
\|\mathbf{u}\|_{1} & \leq \frac{\|\mathbf{f}\|_{-1}+C_{1}\left\|\mathbf{g}_{\mu}\right\|_{1}+C_{2}\left\|\mathbf{g}_{\mu}\right\|_{1}^{2}}{\varepsilon_{0} / \operatorname{Re}-C_{3}\|\beta\|_{0, \infty}\left\|\mathbf{g}_{\mu}\right\|_{1}} \\
& =: C\left(\left\|\mathbf{g}_{\mu}\right\|_{1},\|\mathbf{f}\|_{-1}\right) .
\end{aligned}
$$

Putting (73), (74), (75), and (77) into (72) and using the inequality

$$
\|\mathbf{f}\|_{-1}\left\|\mathbf{u}_{1}-\mathbf{u}_{2}\right\|_{1} \leq \frac{\varepsilon_{0}}{4 \operatorname{Re}}\left\|\mathbf{u}_{1}-\mathbf{u}_{2}\right\|_{1}^{2}+\frac{2 \operatorname{Re}}{\varepsilon_{0}}\|\mathbf{f}\|_{-1}^{2},
$$

we obtain

$$
\begin{aligned}
0 \geq & \frac{\varepsilon_{0}}{2 \operatorname{Re}}\left\|\mathbf{u}_{1}-\mathbf{u}_{2}\right\|_{1}^{2}-\frac{2 \operatorname{Re}}{\varepsilon_{0}}\|\mathbf{f}\|_{-1}^{2} \\
& -C\left(\left\|\mathbf{g}_{\mu}\right\|_{1},\|\mathbf{f}\|_{-1}\right)\|\beta\|_{0, \infty}\left\|\mathbf{u}_{1}-\mathbf{u}_{2}\right\|_{1}^{2} \\
& -\frac{\varepsilon_{0}}{4 \operatorname{Re}}\left\|\mathbf{u}_{1}-\mathbf{u}_{2}\right\|_{1}^{2}-C\left(\left\|\mathbf{g}_{\mu}\right\|_{1},\|\mathbf{f}\|_{-1}\right)\left\|\mathbf{u}_{1}-\mathbf{u}_{2}\right\|_{1}^{2} .
\end{aligned}
$$

For sufficiently small $\left\|\mathbf{g}_{\mu}\right\|_{1},\|\mathbf{f}\|_{-1}$ the constant $C\left(\left\|\mathbf{g}_{\mu}\right\|_{1},\|\mathbf{f}\|_{-1}\right)$ in (77) gets small and consequently the right hand side of (79) is nonnegative. This implies $\mathbf{u}_{1}=\mathbf{u}_{2}$ and according to Theorem $9 p_{1}-p_{2}=0$.

\section{A Channel Flow Problem in Packed Bed Reactors}

In this section, we provide an example of the flow problem in packed bed reactors with numerical solutions at small and relatively large Reynolds numbers to show the nonlinear behavior of the velocity solutions. Our numerical tests were conducted using the noncommercial object-oriented finite element package MoonMD [23] that was originally developed by the research group in Magdeburg and used for several benchmarks. The numerical results generated by MoonMD have been also verified by commercial software packages FLUENT $^{\circledR}$ and FEMLAB ${ }^{\circledR}$; see [24] and [3, Chapter 2.6].

Let the reactor channel with Newtonian-fluid be represented by the plain domain $\Omega=(0, L) \times(-R, R)$ where $R=5$ and $L=60$. In all computations we use the porosity distribution from [3, Section 2.2] which is determined experimentally and takes into account the effect of wall channeling in packed bed reactors

$$
\varepsilon(x, y)=\varepsilon(y)=\varepsilon_{\infty}\left\{1+\frac{1-\varepsilon_{\infty}}{\varepsilon_{\infty}} e^{-6(R-|y|)}\right\},
$$

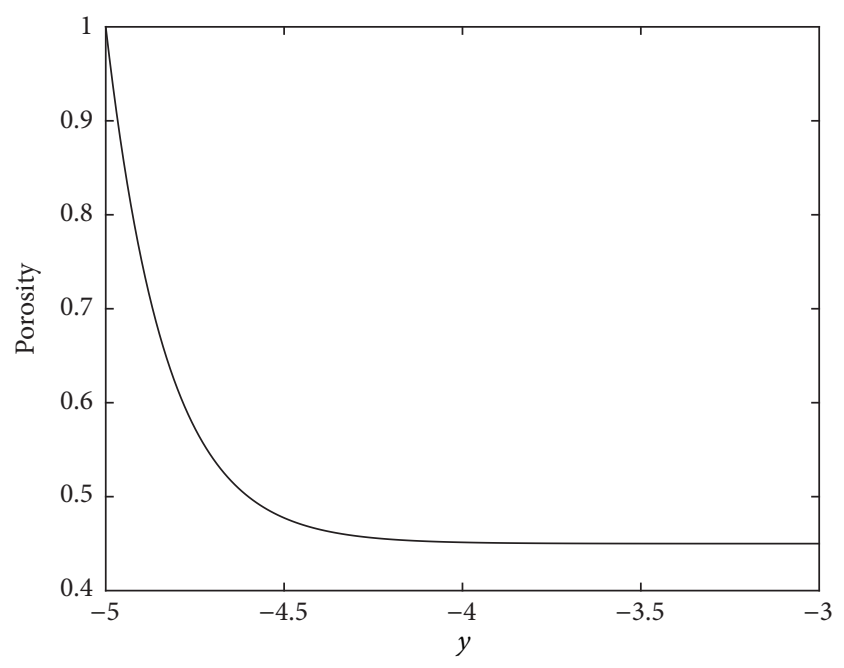

FIGURE 1: Varying porosity.

where $\varepsilon_{\infty}=0.45$. The distribution of the porosity is presented in Figure 1. We distinguish between the inlet, outlet, and membrane parts of domain boundary $\Gamma$ and denote them by $\Gamma_{\text {in }}, \Gamma_{\text {out }}$, and $\Gamma_{w}$, respectively. Let

$$
\begin{aligned}
\Gamma_{\text {in }} & =\{(x, y) \in \Gamma: x=0\}, \\
\Gamma_{\text {out }} & =\{(x, y) \in \Gamma: x=L\}, \\
\Gamma_{w} & =\{(x, y) \in \Gamma: y=-R, y=R\} .
\end{aligned}
$$

At the inlet $\Gamma_{\text {in }}$ and at the membrane wall $\Gamma_{w}$ we prescribe Dirichlet boundary conditions, namely, the plug flow conditions

$$
\begin{aligned}
& \left.\mathbf{u}\right|_{\Gamma_{\text {in }}}=\mathbf{u}_{\text {in }}=\left(u_{\text {in }}, 0\right)^{T}, \\
& \mathbf{u}_{\Gamma_{w}}=\mathbf{u}_{w}= \begin{cases}\left(0, u_{w}\right)^{T} & \text { for } y=-R, \\
\left(0,-u_{w}\right)^{T} & \text { for } y=R,\end{cases}
\end{aligned}
$$

where $u_{\text {in }}>0$ and $u_{w} \geq 0$. We consider the case of fixed bed reactor, that is, $u_{w}=0$. At the outlet $\Gamma_{\text {out }}$ we set the following outflow boundary condition:

$$
-\frac{1}{\operatorname{Re}} \frac{\partial \mathbf{u}}{\partial \mathbf{n}}+p \mathbf{n}=\mathbf{0}
$$

where $\mathbf{n}$ denotes the outer normal. This boundary condition results from the integration by parts when deriving the weak formulation, and it is called the do-nothing boundary condition. In order to avoid discontinuity between the inflow and wall conditions we replace constant profile by trapezoidal one with zero value at the corners. Our computations are carried out on the Cartesian mesh which consists of 100 stretched rectangular cells on the coarse level (see Figure 2) and will be three times uniformly refined. In order to approximate the weak solution of the dimensionless system from (6) subject to the Dirichlet and do-nothing boundary conditions, we apply biquadratic conforming and discontinuous piecewise linear finite elements for the velocity and pressure, respectively. 


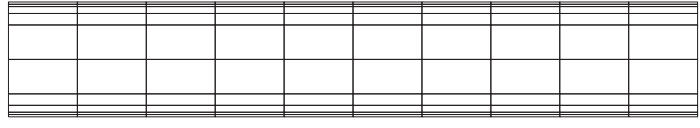

Figure 2: Initial mesh for reactor flow problem.

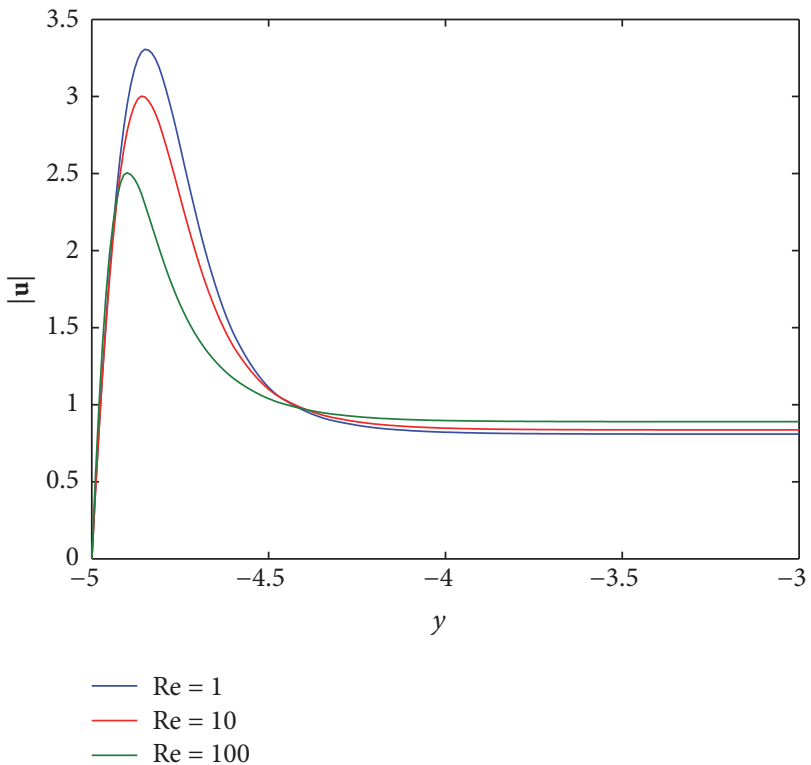

FIgURE 3: Flow profiles in fixed bed reactor at $x=50$.

The detailed finite element analysis of the BrinkmanForchheimer-Darcy equation will be presented in the forthcoming work. The plots of velocity magnitude in fixed bed reactor $\left(u_{w}=0\right)$ are presented along the vertical axis $x=50$. In the investigated reactor the inlet velocity is assumed to be normalized $\left(u_{\text {in }}=1\right)$. Due to the variation of porosity we might expect higher velocity at the reactor walls $\Gamma_{w}$. This tunneling effect can be well observed in Figure 3 which shows the velocity profiles for different Reynolds numbers. This effect is not present when applying standard Brinkman equation with constant porosity. We remark that the maximum of velocity magnitude decreases with increasing Reynolds numbers in the considered cases $\operatorname{Re}=1,10,100$. Our preliminary results are comparable with those ones obtained in [3, Chapter 4.3]. The weighted areas under each velocity profile $u=u(y)$ are the same due to conservation of mass. In fact, we have according to the divergence theorem

$$
\begin{aligned}
\int_{(0,50) \times(-R, R)} \operatorname{div}(\varepsilon \mathbf{u}) d \mathbf{x} & =0 \Longrightarrow \\
\int_{-R}^{R} \varepsilon(y) u(y) d y & =\int_{-R}^{R} \varepsilon(y) v_{\mathrm{in}} d y .
\end{aligned}
$$

However, we expect increasing maximum of the velocity in the case of high Reynolds numbers. This case requires stabilized finite element methods and will be considered in the forthcoming works.

\section{Conclusion}

In this work, we have extended the existence and uniqueness of solution result in literature for the porous medium flow problem based on the nonlinear Brinkman-Forchheimerextended Darcy law. The existing result is valid only for constant porosity and without the considered convection effects, and our result holds for variable porosity and it includes convective effects. We also provided a numerical solution to demonstrate the nonlinear velocity solutions at moderately large Reynolds numbers for which case the Brinkman-Forchheimer-Darcy law applies.

\section{Conflicts of Interest}

The authors declare that there are no conflicts of interest regarding the publication of this paper.

\section{References}

[1] M. g. Peszynska, A. Trykozko, and W. Sobieski, "Forchheimer law in computational and experimental studies of flow through porous media at porescale and mesoscale," in Current advances in nonlinear analysis and related topics, vol. 32 of GAKUTO Internat. Ser. Math. Sci. Appl., pp. 463-482, Gakkotosho, Tokyo, 2010.

[2] S. Ergun, "Fluid flow through packed columns," Chemical Engineering Progress, vol. 48, no. 2, pp. 89-94, 1952.

[3] A. Seidel-Morgenstern, Ed., Membrane Reactors: Distributing Reactants to Improve Selectivity and Yield, Wiley-VCH, 2010.

[4] O. Bey, Strömungsverteilung und Wärmetransport in Schüttungen Nummer 570 in Fortschritt-Berichte, vol. 3, VDI Verlag, Düsseldorf, Germany, 1998.

[5] K. Vafai and S. J. Kim, "On the limitations of the BrinkmanForchheimer-extended Darcy equation," International Journal of Heat and Fluid Flow, vol. 16, no. 1, pp. 11-15, 1995.

[6] U. Hornung, Homogenization and Porous Media, Springer, New York, NY, USA, 1997.

[7] C. R. Garibotti and M. Peszyńska, "Upscaling non-Darcy flow," Transport in Porous Media, vol. 80, no. 3, pp. 401-430, 2009.

[8] V. Matossian, V. Bhat, M. Parashar et al., "Autonomie oil reservoir optimization on the grid," Concurrency Computation Practice and Experience, vol. 17, no. 1, pp. 1-26, 2005.

[9] M. Winterberg and E. Tsotsas, "Modelling of heat transport in beds packed with spherical particles for various bed geometries and/or thermal boundary conditions," International Journal of Thermal Sciences, vol. 39, no. 5, pp. 556-570, 2000.

[10] D. A. Nield and A. Bejan, Convection in Porous Media, Springer, New York, NY, USA, 2nd edition, 1999.

[11] T. Zhao, Investigation of Landslide-Induced Debris Flows by the DEM and CFD [Ph.D. thesis], University of Oxford, 2014.

[12] K. Upton, Multi-scale modelling of borehole yields in chalk aquifers [Ph.D. thesis], Imperial College London, 2015.

[13] A. Grillo, M. Carfagnay, and S. Federicoz, "The DarcyForchheimer law for modelling fluid flow in biological tissues," Theoretical and Applied Mechanics, vol. 41, no. 4, pp. 283-322, 2014.

[14] W. Sobieski and A. Trykozko, "Darcy's and Forchheimer's law in practice. Part 1. the experiment," Technical Sciences, vol. 17, no. 4, pp. 321-335, 2014. 
[15] A. S. Lal and A. C. Menon, "Design of a new porous medium heat exchanger for an aircraft refrigeration system," IJTEL, vol. 3, pp. 545-548, 2014.

[16] P. N. Kaloni and J. Guo, "Steady nonlinear double-diffusive convection in a porous medium based upon the BrinkmanForchheimer model," Journal of Mathematical Analysis and Applications, vol. 204, no. 1, pp. 138-155, 1996.

[17] P. S. Vassilevski and U. Villa, "A mixed formulation for the Brinkman problem," SIAM Journal on Numerical Analysis, vol. 52, no. 1, pp. 258-281, 2014.

[18] C. Bernardi, F. Laval, B. Metivet, and B. Pernaud-Thomas, "Finite element approximation of viscous flows with varying density," SIAM Journal on Numerical Analysis, vol. 29, no. 5, pp. 1203-1243, 1992.

[19] V. Girault and P. Raviart, Finite Element Methods for NavierStokes Equations, Theory and Algorithms, vol. 5 of Springer Series in Computational Mathematics, Springer, Berlin, Germany, 1986.

[20] R. A. Adams, Sobolev Spaces, Pure and Applied Mathematics, Academic Press, New York, NY, USA, 1995.

[21] E. Hopf, "Ein allgemeiner Endlichkeitssatz der Hydrodynamik," Mathematische Annalen, vol. 117, pp. 764-775, 1941.

[22] J. L. Lions, Quelques Méthodes de Résolution des Problèmes aux Limites Non Linéaires, Dunod, Paris, Farnce, 1969.

[23] V. John and G. Matthies, "MooNMD—a program package based on mapped finite element methods," Computing and Visualization in Science, vol. 6, no. 2-3, pp. 163-169, 2004.

[24] A. Tota, K. Georgieva, M. Mangold, and P. Skrzypacz, "Comparative numerical simulations of a packed bed membrane reactor," in Proceedings of the 6th International Conference on Catalysis in Membrane Reactor (ICCMR-6 '04), Lahnstein, Germany, 2004. 


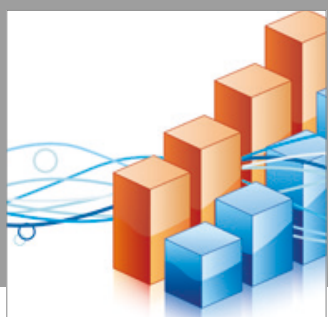

Advances in

Operations Research

vatersals

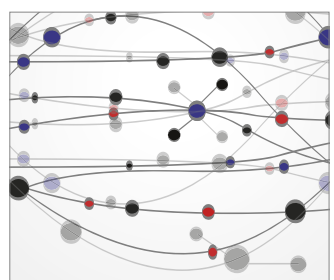

\section{The Scientific} World Journal
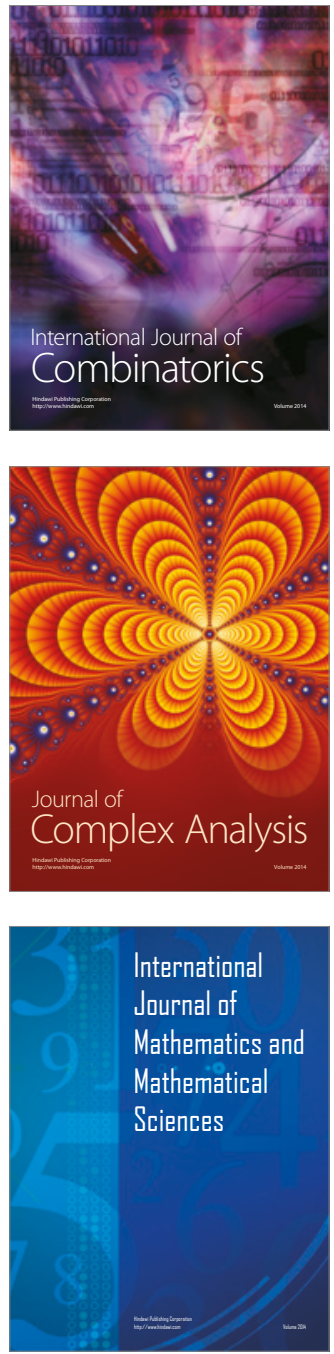
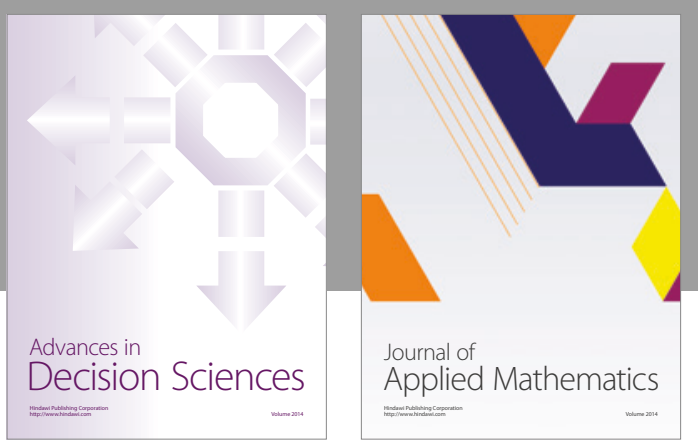

Algebra

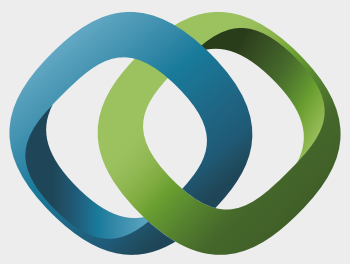

\section{Hindawi}

Submit your manuscripts at

https://www.hindawi.com
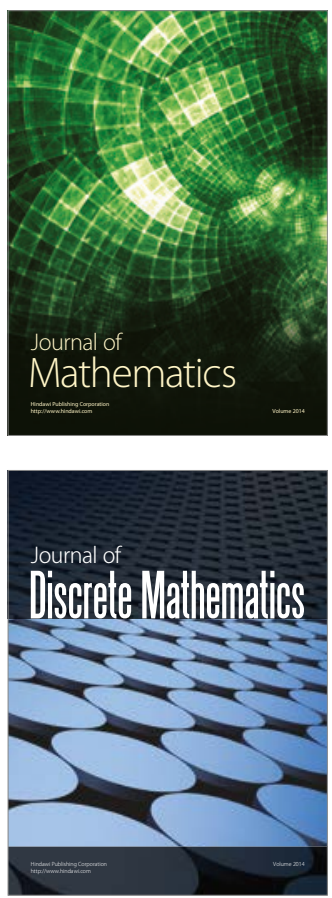

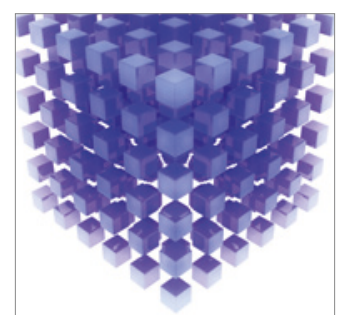

Mathematical Problems in Engineering
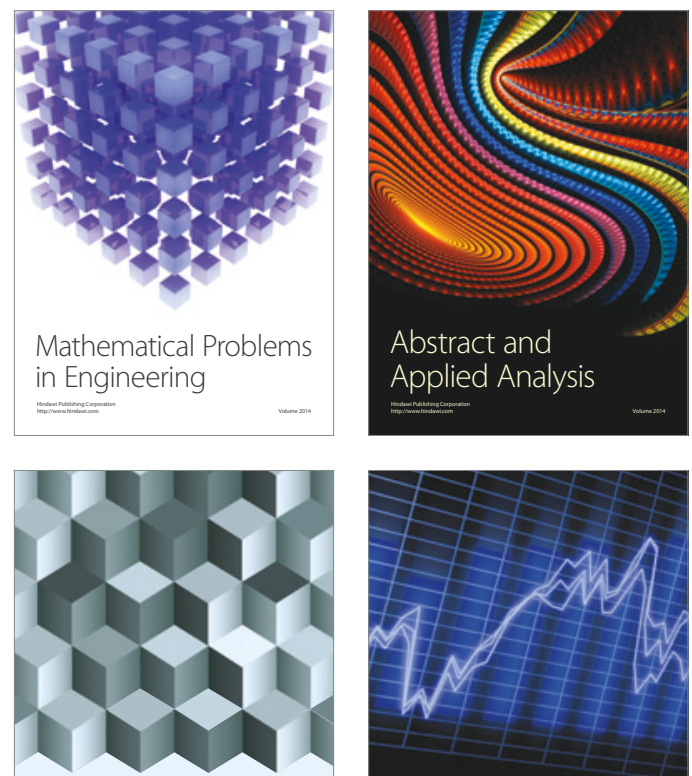

Journal of

Function Spaces

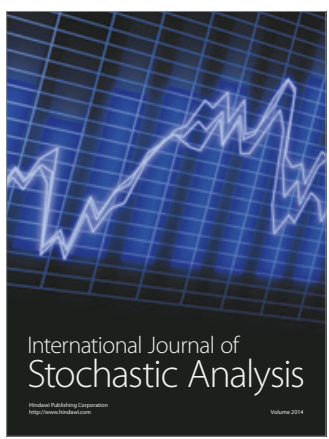

Probability and Statistics
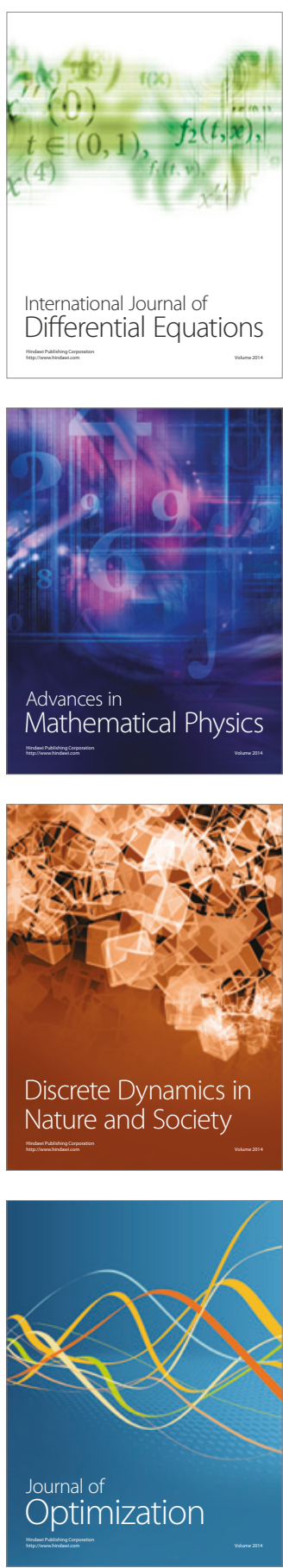